Pacific Journal of Mathematics

BOUNDS AND QUANTITATIVE COMPARISON THEOREMS
FOR NONOSCILLATORY SECOND ORDER DIFFERENTIAL

Mas THornton Read 


\title{
BOUNDS AND QUANTITATIVE COMPARISON THEOREMS FOR NONOSCILLATORY SECOND ORDER DIFFERENTIAL EQUATIONS
}

\author{
Thomas T. READ
}

\begin{abstract}
A lower bound is given for the positive increasing solution of $y^{\prime \prime}+2 r y^{\prime}-q^{2} y=0$ on the interval $[0, \infty)$ and an upper bound is given for the positive decreasing solution of this equation. These are used to estimate $z / y$ and $z_{0} / y_{0}$ where $y$ and $z$ (respectively $y_{0}$ and $z_{0}$ ) are positive nonprincipal (respectively principal) solutions of nonoscillatory equations $y^{\prime \prime}-p_{1} y=0$ and $z^{\prime \prime}-p_{2} z=0$ for which $p_{2} \geqq p_{1}$. A special case of one result is that if $p_{1}$ is bounded and if

$$
\lim \inf \left(\int_{0}^{x}\left(p_{2}-p_{1}\right)^{1 / 2} d t / x\right)>0
$$

as $x \rightarrow \infty$ then $z / y$ increases exponentially and $z_{0} / y_{0}$ decreases exponentially.
\end{abstract}

1. Introduction. It is our objective to estimate the relative sizes of the solutions of two nonoscillatory differential equations

$$
y^{\prime \prime}-p_{1} y=0
$$

and

$$
z^{\prime \prime}-p_{2} z=0
$$

on the interval $[0, \infty)$ when $p_{2} \geqq p_{1}$.

It is well-known that (1) has a unique, principal, solution $y_{0}$ with the property that for any linearly independent, nonprincipal, solution $y, \lim y_{0}(x) / y(x)=0$ as $x \rightarrow \infty$. If $p_{1}$ is nonnegative, then $y_{0}$ may be taken to be positive and decreasing and there is a nonprincipal solution which is positive and increasing [3, p. 355].

Our first task will be to obtain a lower bound for such a nonprincipal solution and an upper bound for the principal solution. We shall actually do this in $\S \S 2$ and 3 for the more general equation

$$
y^{\prime \prime}+2 r y^{\prime}-q^{2} y=0 \text {. }
$$

Here $r$ and $q$ are required only to be real-valued and locally integrable. Note, however, from the form of (3) that we may also assume that $q$ is nonnegative. The estimates are in terms of $\int_{0}^{x} r d t, \int_{0}^{x} q d t$, and an increasing function $F$. In the special case when $r=0$ they take the form that if $\lim \inf \left(\int_{0}^{x} q d t / F(x)\right)>1$ as $x \rightarrow \infty$ for suitable $F$, 
then there is an increasing solution $y$ such that $y(x) \geqq y\left(x_{0}\right) e^{F(x)}$ for $x \geqq x_{0}$ and a positive decreasing solution $y_{0}$ such that $y_{0}(x) \leqq y_{0}\left(x_{0}\right) e^{-F(x)}$ for $x \geqq x_{0}$. The case $F(x)=c x$ of this result was discussed in [4].

For sufficiently smooth $r$ and $q$ satisfying appropriate conditions, asymptotic formulae have been given for the solutions of (3). (See, for instance, [1, p. 120].) Our estimates, however, require no assumptions on $r$ and $q$ beyond local integrability and are thus available when the asymptotic formulae are not. One such application, to the deficiency index theory of powers of formally symmetric nonoscillatory expressions, is given in [5] where it is shown for $M(y)=$ $-\left(p y^{\prime}\right)^{\prime}+q y$ that if $q$ is nonnegative and if $\int_{0}^{x}(q / p)^{1 / 2} d t \geqq K \log p(x)$ for some $K>[n(n-1)]^{1 / 2}$ and all $x$ in a set of infinite measure, then $M^{j}$ is limit-point for $j=1,2, \cdots, n$.

Here we apply these estimates in a different direction. It is essentially a form of Sturm's comparison theorem that each pair of eventually positive nonprincipal solutions $y$ and $z$ of (1) and (2) respectively satisfies $z^{\prime} / z \geqq y^{\prime} / y$ on some interval $[a, \infty)$ and that the eventually positive principal solutions $y_{0}$ and $z_{0}$ satisfy $z_{0}^{\prime} / z_{0} \leqq$ $y_{0}^{\prime} / y_{0}$ on $[a, \infty)[3$, p. 359]. Hence for all sufficiently large $x, z / y$ is a positive increasing function and $z_{0} / y_{0}$ is a positive decreasing function.

In $\S 5$ we shall use our earlier estimates to give a lower bound for $z / y$ and an upper bound for $z_{0} / y_{0}$ in terms of $p_{2}-p_{1}$. A special case of our result is that if $p_{1}$ is bounded and if

$$
\lim \inf \left(\int_{0}^{x}\left(p_{2}-p_{1}\right)^{1 / 2} d t / x\right)>0
$$

as $x \rightarrow \infty$, then $z / y$ increases exponentially and $z_{0} / y_{0}$ decreases exponentially.

In the general result the boundedness of $p_{1}$ is replaced by a growth condition on $\int_{0}^{x} p_{1} d t$. We show that such a condition cannot be omitted by proving for nonnegative $p_{1}$ and $p_{2}$ that if $p_{2}-p_{1}$ is bounded and $p_{2}^{1 / 2}-p_{1}^{1 / 2}$ approaches 0 , then $z / y$ increases more slowly than any exponential. In particular, this conclusion holds for certain functions $p_{1}$ and $p_{2}$ for which $p_{2}-p_{1}$ is bounded away from 0 .

In $\S 4$ we shall derive an upper bound for the nonprincipal solution of (1) and a lower bound for the principal solution. These depend on $\int_{0}^{x}\left|p_{1}\right| d t$ rather than $\int_{0}^{x}\left|p_{1}\right|^{1 / 2} d t$ as one might hope from the other bounds. We give an example to show that this cannot be avoided.

2. A lower bound for the nonprincipal solution. We begin by deriving a lower bound for an increasing solution of (3) valid on 
the complement of a set of finite Lebesgue measure.

THEOREM 2.1. Let $r$ and $q$ be real-valued locally integrable functions on $[0, \infty)$ with $q$ nonnegative. Let $y$ be any solution of (3) with $y(0)>0$ and $y^{\prime}(0)>0$. Then for each $c, 0<c<1$, there is a subset $E_{\mathrm{c}}$ of $[0, \infty)$ such that $m\left(E_{\mathrm{c}}\right) \leqq y(0) c^{2} / y^{\prime}(0)\left(1-c^{2}\right)$ and for all $x$ not in $E_{c}$,

$$
y(x) \geqq y(0) \exp c\left(\left[c^{2}\left(\int_{0}^{x} r d t\right)^{2}+\left(\int_{0}^{x} q d t\right)^{2}\right]^{1 / 2}-c \int_{0}^{x} r d t\right) .
$$

Proof. Let $y$ be as in the statement of the theorem. Set $f(x)=\exp 2 \int_{0}^{x} r d t$ and $w=f y^{\prime} / y$. Note that

$$
\log y(x)=\log y(0)+\int_{0}^{x} w / f d t
$$

$w$ is positive since $y$ is increasing and satisfies the Riccati equation $w^{\prime}=q^{2} f-w^{2} / f$ or, equivalently,

$$
w^{\prime} / w+w / f=q^{2} f / w .
$$

From Schwarz's inequality and (5),

$$
\begin{aligned}
\left(\int_{0}^{x} q d t\right)^{2} & \leqq \int_{0}^{x} w / f d t \int_{0}^{x} q^{2} f / w d t \\
& \leqq \int_{0}^{x} w / f d t\left[\int_{0}^{x} w / f d t+\log (w(x) / w(0) f(x))+2 \int_{0}^{x} r d t\right]
\end{aligned}
$$

We shall complete the proof by showing that $\log (w(x) / w(0) f(x))$ is small compared to $\int_{0}^{x} w / f d t$ off a set of finite measure. Fix $c<1$ and set $b=\left(1-c^{2}\right) / c^{2}$. Let $E_{c}$ denote the set of all $x$ for which $\log (w(x) / w(0) f(x)) \geqq b \int_{0}^{x} w / f d t$, that is for which

$$
W(x)=[w(x) / f(x)] \exp \left(-b \int_{0}^{x} w / f d t\right) \geqq w(0) .
$$

Since $W$ is positive and is the derivative of $(-1 / b) \exp \left(-b \int_{0}^{x} w / f d t\right)$,

$$
1 / b \geqq \int_{0}^{\infty} W d x \geqq \int_{E_{\mathrm{c}}} w(0) d x=w(0) m\left(E_{\mathrm{c}}\right) .
$$

Recalling the definitions of $b$ and $w$,

$$
m\left(E_{c}\right) \leqq y(0) c^{2} / y^{\prime}(0)\left(1-c^{2}\right) .
$$

For $x \notin E_{c}$ we have from (6) that 


$$
\left(\int_{0}^{x} q d t\right)^{2} \leqq \int_{0}^{x} w / f d t\left[\left(1 / c^{2}\right) \int_{0}^{x} w / f d t+2 \int_{0}^{x} r b d t\right] .
$$

The conclusion of Theorem 2.1 now follows by solving this inequality for $\int_{0}^{x} w / f d t$ and substituting the result into (4).

It should be noted that the exceptional sets $E_{c}$ can be unbounded and of positive measure for each $c>0$. An example with $r=0$ for which this occurs is given in [4].

From the basic estimate we can now quickly obtain a lower bound for nonprincipal solutions of (3).

THEOREM 2.2. Let $r$ and $q$ be as in Theorem 2.1. Let $F$ be an increasing differentiable function on $[0, \infty)$ such that $F^{\prime \prime} \leqq K F$ for some positive constant $K$. If

$$
\liminf _{x \rightarrow \infty}\left(\left[\left(\int_{0}^{x} q d t\right)^{2}+\left(\int_{0}^{x} r d t\right)^{2}\right]^{1 / 2}-\int_{0}^{x} r d t\right) / F(x)>1
$$

then for any increasing solution $y$ of (3),

$$
y(x) \geqq y(0) e^{F(x)}
$$

for all $x$ greater than some $x_{0}$.

Proof. Choose $\delta>0$ and $x_{1}$ so that the expression in (7) is greater than $1+\delta$ for $x \geqq x_{1}$. Choose $c<1$ so that $c^{2}(1+\delta)=1+\delta^{\prime}>1$. Let $E_{c}$ be as in Theorem 2.1. Then for $x$ greater than $x_{1}$ and not in $E_{c}$,

$$
\begin{aligned}
\log y(x) & \geqq \log y(0)+c\left(\left[\left(\int_{0}^{x} q d t\right)^{2}+c^{2}\left(\int_{0}^{x} r d t\right)^{2}\right]^{1 / 2}-c \int_{0}^{x} r d t\right) \\
& \geqq \log y(0)+c^{2}\left(\left[\left(\int_{0}^{x} q d t\right)^{2}+\left(\int_{0}^{x} r d t\right)^{2}\right]^{1 / 2}-\int_{0}^{x} r d t\right) \\
& \geqq \log y(0)+\left(1+\delta^{\prime}\right) F(x) .
\end{aligned}
$$

Now choose $\varepsilon<\delta^{\prime} / K\left(1+\delta^{\prime}\right)$ and $x_{2}$ so that $m\left(E_{c} \cap\left[x_{2}, \infty\right)\right)<\varepsilon$. Then for any $x \geqq x_{0}=\max \left(x_{1}, x_{2}\right)$ there exists $X \in[x-\varepsilon, x]$ such that

$$
\begin{aligned}
\log y(x) & \geqq \log y(X) \geqq \log y(0)+\left(1+\delta^{\prime}\right) F(X) \\
& =\log y(0)+F(x)+\left(1+\delta^{\prime}\right)(F(X)-F(x))+\delta^{\prime} F(x) \\
& \geqq \log y(0)+F(x) .
\end{aligned}
$$

The last inequality follows from the Mean Value Theorem and the choice of $\delta$. This completes the proof.

In particular we have the following criterion for exponential growth. 
COROLlaRY 2.3. Let $r$ and $q$ be as in Theorem 2.1. If for some $\alpha>0$

$$
\liminf _{x \rightarrow \infty}\left(\left[\left(\int_{0}^{x} q d t\right)^{2}+\left(\int_{0}^{x} r d t\right)^{2}\right]^{1 / 2}-\int_{0}^{x} r d t\right) / x>\alpha,
$$

then for any increasing solution y of (3),

$$
y(x) \geqq y(0) e^{\alpha x}
$$

for all $x$ greater than some $x_{0}$.

It is clear from the constant coefficient case that the exponent is the best possible.

3. An upper bound for the principal solution. We now take up the problem of finding an upper bound for the principal solution of (3). Our method will be slightly different than in the previous section; instead of deriving a general inequality like Theorem 2.1 we shall proceed directly to an analog of Theorem 2.2. However the proof, in part, is similar to the proof of Theorem 2.1. It is convenient to begin with a lemma.

LEMMA 3.1. If $u$ and $v$ are locally integrable functions on $[0, \infty)$ with $v$ nonnegative, $u$ positive and $u^{-\alpha} \in L_{1}(0, \infty)$ for all $0<\alpha<1 / 2$, and if

$$
u(x) \geqq K \exp \int_{0}^{x} u v d t
$$

for all sufficiently large $x$ and some positive $K$, then $v^{\beta} \in L_{1}(0, \infty)$ for all $0<\beta \leqq 1$.

Proof. For any nonnegative $u$ and $v$ and any positive $\gamma$,

$$
u v \exp \left(-\gamma \int_{0}^{x} u v d t\right)=(-1 / \gamma)\left[\exp \left(-\gamma \int_{0}^{x} u v d t\right)\right]^{\prime} \in L_{1}(0, \infty) \text {. }
$$

We have here that eventually $v \leqq K^{-1} u v \exp \left(-\int_{0}^{x} u v d t\right)$. Hence $v \in L_{1}(0, \infty)$. Let $E$ be a set of finite measure such that for all $x$ in the complement, $D$, of $E$, the hypothesis of the lemma holds and also $u v \exp \left(-1 / 2 \int_{0}^{x} u v d t\right) \leqq K^{1 / 2}$. Thus on $D$,

$$
u(x) \geqq K \exp \int_{0}^{x} u v d t \geqq u^{2}(x) v^{2}(x) .
$$

Then for any $0<\beta<1, u^{-\beta / 2} \geqq v^{\beta}$ on $D$ and so $\int_{D} v^{\beta} d t<\infty$. 
Now write $E$ as the disjoint union $E=A \cup B$ where $A=$ $\{x \in E: v(x) \leqq 1\}$. Clearly $\int_{A} v^{\beta} d t<\infty$. For any $\beta<1$ we have also $\int_{B} v^{\beta} d t \leqq \int_{B} v d t<\infty$. Collecting the results on $A, B$ and $D$, the lemma is proved.

THEOREM 3.2. Let $r$ and $q$ be as in Theorem 2.1. Let $F$ be an increasing differentiable function such that $F^{\prime \prime} e^{\alpha F} \rightarrow \infty$ as $x \rightarrow \infty$ for each positive $\alpha$. If

$$
\liminf _{x \rightarrow \infty}\left(\left[\left(\int_{0}^{x} q d t\right)^{2}+\left(\int_{0}^{x} r d t\right)^{2}\right]^{1 / 2}+\int_{0}^{x} r d t\right) / F(x)>1
$$

then for the positive decreasing solution $y_{0}$ of (3),

$$
y_{0}(x) \leqq y_{0}(0) e^{-F(x)}
$$

for all $x$ greater than some $x_{0}$.

Proof. We shall for the first part of the proof assume in addition that $q \notin L_{1}(0, \infty)$. Set $f(x)=\exp 2 \int_{0}^{x} r d t$ and $w=-f y_{0}^{\prime} / y_{0}$. Then $w$ is positive and $w^{\prime}=w^{2} / f-q^{2} f$. Also

$$
\log y_{0}(x)=\log y_{0}(0)-\int_{0}^{x} w / f d t
$$

so that we must show $\int_{0}^{x} w / f d t \geqq F(x)$ for $x \geqq x_{0}$.

We assert first that $\int_{0}^{x} w / f d t \geqq F(x)$ for arbitrarily large values of $x$. A calculation using Schwarz's lemma as in the proof of Theorem 2.1 yields

$$
\begin{aligned}
\left(\int_{0}^{x} q d t\right)^{2} \leqq & \int_{0}^{x} w / f d t\left[\int_{0}^{x} w / f d t-2 \int_{0}^{x} r d t\right. \\
& +\log (f(x) w(0) / w(x))] .
\end{aligned}
$$

Choose $\delta>0$ and $x_{1}$ so that the expression in (8) is greater than $1+\delta$ for all $x \geqq x_{1}$. Suppose that for some $x_{2} \geqq x_{1}, \int_{0}^{x} w / f d t<F(x)$ for all $x \geqq x_{2}$. Then for $x \geqq x_{2}$,

$$
\left(\int_{0}^{x} q d t\right)^{2} \leqq F(x)\left[F(x)-2 \int_{0}^{x} r d t+\log (f(x) w(0) / w(x))\right] .
$$

In order to complete the proof of the assertion we need the fact that for any real numbers $A, B$, and $F$ with $F$ nonnegative, if $\left(A^{2}+B^{2}\right)^{1 / 2}+B \geqq(1+\delta) F$, then $A^{2}+2 B F-F^{2} \geqq \delta F^{2}$. This is clear if $2 B \geqq(1+\delta) F$. Otherwise, let $B=\varepsilon F$ with $2 \varepsilon<1+\delta$. Then $A^{2}+B^{2} \geqq(1+\delta-\varepsilon)^{2} F^{2}$ so that 


$$
\begin{aligned}
A^{2}+2 B F & \geqq\left(1+2 \delta+\delta^{2}-2 \delta \varepsilon\right) F^{2} \\
& =[1+\delta+\delta(1+\delta-2 \varepsilon)] F^{2} \\
& \geqq(1+\delta) F^{2} .
\end{aligned}
$$

For this inequality and (8) we have that

$$
\left(\int_{0}^{x} q d t\right)^{2}+2 F(x) \int_{0}^{x} r d t-F^{2}(x) \geqq \delta F^{2}(x)
$$

for all $x \geqq x_{1}$. Thus from (10) we obtain that for $x \geqq x_{2}$,

$$
\begin{gathered}
\log (f(x) w(0) / w(x)) \geqq \delta F(x) \quad \text { or } \\
f(x) / w(x) \geqq e^{\delta F^{\prime}(x)} / w(0) .
\end{gathered}
$$

To see that (11) cannot hold for all $x \geqq x_{2}$, set $u=f / w$. Then $u$ is positive and

$$
u^{\prime} / u-2 r+u^{-1}=u q^{2} .
$$

It follows from (8) that $\left(\int_{0}^{x} q d t\right)^{2} \geqq-2 F(x) \int_{0}^{x} r d t$ for all $x \geqq x_{1}$. By integrating (12) from 0 to $x$ and using this inequality we obtain

$$
\log (u(x) / u(0))+\left(\int_{0}^{x} q d t\right)^{2} / F(x)+\int_{0}^{x} u^{-1} d t \geqq \int_{0}^{x} u q^{2} d t .
$$

For all $x$ greater than some $x_{3}, F^{\prime}(x) e^{\delta F(x)} \geqq 3 w(0)$ and hence $u^{-1}(x) \leqq w(0) e^{-\delta F(x)} \leqq F^{\prime}(x) / 3$. Thus by the Schwarz inequality,

$$
\begin{aligned}
\left(\int_{x_{3}}^{x} q d t\right)^{2} & \leqq \int_{x_{3}}^{x} u^{-1} d t \int_{x_{3}}^{x} u q^{2} d t \leqq \frac{1}{3}\left[F(x)-F\left(x_{3}\right)\right] \int_{x_{3}}^{x} u q^{2} d t \\
& \leqq \frac{1}{3} F(x) \int_{0}^{x} u q^{2} d t .
\end{aligned}
$$

Hence, since $q \notin L_{1}(0, \infty)$,

$$
\int_{0}^{x} u q^{2} d t \geqq 2\left(\int_{0}^{x} q d t\right)^{2} / F(x)
$$

for all sufficiently large $x$.

For such $x$,

$$
\log (u(x) / u(0))+\int_{0}^{x} u^{-1} d t \geqq \frac{1}{2} \int_{0}^{x} u q^{2} d t .
$$

Moreover, $u^{-\alpha} \in L_{1}(0, \infty)$ for each $\alpha>0$, since for all sufficiently large $x$ we have from (11) and the assumption on $F$ that

$$
u^{-\alpha}(x) \leqq C e^{-\alpha \delta F(x)} \leqq F^{\prime}(x) e^{-(\alpha \delta / 2) F(x)} \in L_{1}(0, \infty) .
$$

Hence for all sufficiently large $x$ and some positive $K$, 


$$
u(x) \geqq K \exp \frac{1}{2} \int_{0}^{x} u q^{2} d t .
$$

Now Lemma 3.1 with $\beta=1 / 2$ applied to $u$ and $v=q^{2} / 2$ implies that $q \in L_{1}(0, \infty)$. But this contradicts the extra assumption made at the beginning of the proof. Hence $\int_{0}^{x} w / f d t \geqq F(x)$ for arbitrarily large values of $x$ as asserted.

Now choose $x_{0}$ so that $x \geqq x_{0}$ implies $F^{\prime}(x) \geqq 2 w(0) e^{-\delta F(x)}$. Suppose that for some $X \geqq x_{0}, \int_{0}^{X} w / f d t<F(X)$. Let

$$
X^{\prime}=\inf \left\{x>X: \int_{0}^{x} w / f d t \geqq F(x)\right\} .
$$

On the interval $\left(X, X^{\prime}\right)$ the inequality (11) is valid, that is, $w(x) / f(x) \leqq$ $1 / 2 F^{\prime}(x)$. Hence

$$
\begin{aligned}
\int_{0}^{X^{\prime}} w / f d t= & \int_{0}^{X} w / f d t+\int_{X}^{X^{\prime}} w / f d t<F(X) \\
& +\frac{1}{2} \int_{X}^{X^{\prime}} F^{\prime} d t<F\left(X^{\prime}\right) .
\end{aligned}
$$

This contradiction completes the proof of the theorem when $q \notin L_{1}(0, \infty)$.

Now suppose $q \in L_{1}(0, \infty)$. Then (8) implies that

$$
\liminf _{x \rightarrow \infty} 2 \int_{0}^{x} r d t / F(x)>1 .
$$

Let $z$ be the positive decreasing solution of $z^{\prime \prime}+2 r z^{\prime}=0$ such that $z(0)=1$. Then

$$
z(x)=k \int_{x}^{\infty} \exp \left(-2 \int_{0}^{t} r(u) d u\right) d t .
$$

For all sufficiently large $t$,

$$
k \exp \left(-2 \int_{0}^{t} r(u) d u\right) \leqq k \exp (-(1+\delta) F(t)) \leqq F^{\prime}(t) e^{-F^{\prime}(t)}
$$

so that $z(x) \leqq \int_{x}^{\infty} F^{\prime} e^{-F} d t=e^{-F(x)}$. But the principal solution $y_{0}$ of (3) satisfies $y_{0}(x) \leqq y_{0}(0) z(x)$ [3, p. 359] so that the conclusion is true in this case also and the proof is complete.

The hypothesis $F^{\prime} e^{\alpha F} \rightarrow \infty$ for all $\alpha>0$ is satisfied, for example, for every positive power of $x$. For the function $F(x)=\log (1+x)$, corresponding to solutions of the form $x^{-r}$, it is satisfied only for $\alpha>1$. Examples of the form $y^{\prime \prime}-k(1+x)^{-2} y=0$ for small positive $k$ show that in fact the result is no longer true for this choice of $F$. However a slight modification of the proof of Theorem 3.2 does yield the following result for $q \notin L_{1}(0, \infty)$. 
COROLLARY 3.3. Let $r$ and $q$ be as in Theorem 2.1 with $q \notin$ $L_{1}(0, \infty)$. Let $F$ be an increasing differentiable function such that $F^{\prime} e^{\alpha F} \rightarrow \infty$ as $x \rightarrow \infty$ for all $\alpha$ greater than some $\alpha_{0}$. If (8) holds then

$$
y_{0}(x) \leqq y_{0}(0) e^{-\beta F(x)}
$$

for all $x$ greater than some $x_{0}$ and some $\beta>0$.

As in the previous section we state the condition for exponential decay as a corollary of Theorem 3.2.

CoROLlaRy 3.4. Let $r$ and $q$ be as in Theorem 2.1. If for some $\alpha>0$,

$$
\liminf _{x \rightarrow \infty}\left(\left[\left(\int_{0}^{x} q d t\right)^{2}+\left(\int_{0}^{x} r d t\right)^{2}\right]^{1 / 2}+\int_{0}^{x} r d t\right) / x>\alpha
$$

then for the positive decreasing solution $y_{0}$ of (3),

$$
y_{0}(x) \leqq y_{0}(0) e^{-\alpha x}
$$

then all $x$ greater than some $x_{0}$.

Again it is clear from the constant coefficient case that the exponent is the best possible.

4. Bounds for $y^{\prime \prime}-p y=0$. If $q \in L_{1}(0, \infty)$ then certainly no upper bound for the increasing solution of $y^{\prime \prime}-q^{2} y=0$ can be made from $\exp \int_{0}^{x} q d t$. The following example shows that it does not help to require $q \notin L_{1}(0, \infty)$.

Let $\left\{x_{k}\right\}_{k=0}^{\infty}$ be an increasing sequence with $x_{0}=0$ chosen so that if $q(x)=0$ on each $I_{n}=\left[x_{2 n}, x_{2 n+1}\right)$ and $q(x)=3 n$ on each $J_{n}=\left[x_{2 n+1}, x_{2 n+2}\right)$, then the solution $w$ of $w^{\prime}=q^{2}-w^{2}$ with $w\left(x_{0}\right)=e$ satisfies $w\left(x_{2 n}\right)=e$ and $w\left(x_{2 n+1}\right)=1$ for all $n$. Obviously $x_{2 n+1}-x_{2 n}$ does not depend on $n$. Solving the equations $w^{\prime}=-w^{2}$ and $w^{\prime}=9 n^{2}-w^{2}$ it is found that $\int_{I_{n}} w d t=1$ for each $n$ and that for large $n, x_{2 n+2}-x_{2 n+1}$ is approximately $(e-1) / 9 n^{2}$ so that $\int_{J_{n}} q d t \sim(e-1) / 3 n$. Hence the solution $y(x)=\exp \int_{0}^{x} w d t$ of $y^{\prime \prime}-q^{2} y=0$ increases exponentially while $q \notin L_{1}(0, \infty)$ but $\int_{0}^{x} q d t / x \rightarrow 0$.

To overcome this difficulty we replace $\int_{0}^{x} q d t$ by $\left(x \int_{0}^{x} q^{2} d t\right)^{1 / 2}$. There is no advantage in restricting ourselves to nonnegative coefficients, so we state our result in terms of

$$
y^{\prime \prime}-p y=0 \text {. }
$$


THEOREM 4.1. Let $p$ be a locally integrable function on $[0, \infty)$, not identically 0 , such that (13) is nonoscillatory. Let $G$ be an increasing differentiable function such that $x G^{\prime}(x) / G^{2}(x) \rightarrow 0$ as $x \rightarrow \infty$. If

$$
\limsup _{x \rightarrow \infty} x \int_{0}^{x}|p| d t / G^{2}(x)<1,
$$

then every eventually positive solution $y$ of (13) satisfies

$$
y\left(x_{0}\right) e^{-G(x)} \leqq y(x) \leqq y\left(x_{0}\right) e^{G(x)}
$$

for all $x$ greater than some $x_{0}$.

Proof. By applying the comparison theorem discussed in the introduction to $p_{1}=p, p_{2}=|p|$ it is clear that it suffices to establish (14) for solutions of $y^{\prime \prime}-|p| y=0$.

Let $y$ be a positive increasing solution of this equation, and let $w=y^{\prime} / y$. Then $w^{\prime}+w^{2}=|p|$. Hence, integrating from 0 to $x$ and using the positivity of $w, \int_{0}^{x} w^{2} d t \leqq w(0)+\int_{0}^{x}|p| d t$. If $p \in L_{1}(0, \infty)$ then it is well-known that $x^{-1 / 2} \int_{0}^{x} w d t \rightarrow 0$ as $x \rightarrow \infty$ [2, Th. 223, p. 164]; hence $\int_{0}^{x} w d t / G(x) \rightarrow 0$ and the right hand inequality for (14) follows. If $p \notin L_{1}(0, \infty)$, then an application of Schwarz's inequality gives, for sufficiently large $x_{0}$,

$$
\left(\int_{x_{0}}^{x} w d t\right)^{2} \leqq x\left[w(0)+\int_{x_{0}}^{x}|p| d t\right] \leqq G^{2}(x)
$$

for all $x \geqq x_{0}$ and hence the right hand side of (14) follows again. The left hand side is of course trivial in either case.

Now let $y_{0}$ be a positive decreasing solution of $y^{\prime \prime}-|p| y=0$ and let $w=-y_{0}^{\prime} / y_{0}$. Then $w$ is positive and $-w^{\prime}+w^{2}=|p|$. If $w \in L_{1}(0, \infty)$ then certainly for some $x_{0}, \int_{x_{0}}^{x} w d t \leqq G(x)$ for all $x \geqq x_{0}$ and the left hand side in (14) follows.

If $w \notin L_{1}(0, \infty)$ then for sufficiently large $x_{0}$ and some $\delta<1$, $(1-\delta) \int_{0}^{x_{0}} w d t \geqq 1,2 x G^{\prime}(x)<(1-\delta) G^{2}(x)$ for $x \geqq x_{0}$ and

$$
\left(\int_{0}^{x} w d t\right)^{2} / x \leqq \int_{0}^{x}|p| d t+w(x) \leqq \delta G^{2}(x) / x+w(x)
$$

for $x \geqq x_{0}$. Suppose that for some $x_{1} \geqq x_{0}, \int_{x_{0}}^{x_{1}} w d t>G\left(x_{1}\right)$. If this inequality remains valid for all $x \geqq x_{1}$, then for such $x$ it follows from (15) that $x w(x) \geqq(1-\delta)\left(\int_{0}^{x} w d t\right)^{2}$ and hence 


$$
w(x)\left(\int_{0}^{x} w d t\right)^{-2} \geqq(1-\delta) / x
$$

But this cannot be true for all $x \geqq x_{1}$ since the function on the left is in $L_{1}(0, \infty)$ and the function on the right is not.

Thus let $x_{2}=\inf \left\{x>x_{1}: \int_{x_{0}}^{x} w d t \leqq G(x)\right\}$. On $\left(x_{1}, x_{2}\right)$ we have $w(x) \geqq(1-\delta)\left(\int_{0}^{x} w d t\right)^{2} / x>\int_{0}^{x} w d t / x$ and also $w(x) \geqq(1-\delta) G^{2}(x) / x$. Hence on $\left(x_{1}, x_{2}\right)$,

$$
\begin{aligned}
{\left[\left(\int_{x_{0}}^{x} w d t\right)^{2} / x\right]^{\prime} } & =\left(\int_{x_{0}}^{x} w d t / x\right)\left[2 w(x)-\int_{x_{0}}^{x} w d t / x\right] \\
& \geqq w(x) \int_{x_{0}}^{x} w d t / x \\
& \geqq(1-\delta) G^{3}(x) / x^{2} \geqq\left[G^{2}(x) / x\right]^{\prime} .
\end{aligned}
$$

Then $\left(\int_{x_{0}}^{x_{2}} w d t\right)^{2} / x_{2}-G^{2}\left(x_{2}\right) / x_{2} \geqq\left(\int_{x_{0}}^{x_{1}} w d t\right)^{2} / x_{1}-G^{2}\left(x_{1}\right) / x_{1}>0$, contradicting the choice of $x_{2}$. Hence we must have $\int_{x_{0}}^{x} w d t \leqq G(x)$ for $x \geqq x_{0}$ and the left hand inequality in (14) follows. The right hand inequality is clear for the decreasing solution and so the proof is complete.

5. Quantitative comparison theorems. We shall now combine the results of $\S \S 2,3$ and 4 to obtain some comparison theorems. Thus let $p_{1}$ and $p_{2}$ be locally integrable functions on $[0, \infty)$ with $p_{2} \geqq p_{1}$ such that (1) and (2) are nonoscillatory. We wish to obtain a lower bound for the quotient $z / y$ of positive nonprincipal solutions of (1) and (2) and an upper bound for the quotient $z_{0} / y_{0}$ of the positive principal solutions of (1) and (2).

THEOREM 5.1. Let $G$ and $H$ be increasing differentiable functions such that $x G^{\prime}(x) / G^{2}(x) \rightarrow 0, H^{\prime} e^{\alpha H} \rightarrow \infty$ for all $\alpha>0$ and $H^{\prime} / H$ is bounded. Suppose that

(a) $\lim \sup _{x \rightarrow \infty} x \int_{0}^{x}\left|p_{1}\right| d t / G^{2}(x)<1$

(b) $\lim \inf _{x \rightarrow \infty} \int_{0}^{x}\left(p_{2}-p_{1}\right)^{1 / 2} d t / H(x)>1$.

If $H(x) \geqq K G(x)$ for some positive $K$, then

$$
z(x) / y(x) \geqq e^{\beta H(x)} ; z_{0}(x) / y_{0}(x) \leqq e^{-\beta H(x)}
$$

for some $\beta>0$ and all $x$ greater than some $x_{0}$.

Proof. For the first assertion, set $u=z / y$. Then $u$ satisfies $u^{\prime \prime}+2\left(y^{\prime} / y\right) u^{\prime}-\left(p_{2}-p_{1}\right) u=0$. For all sufficiently large $x$ it follows from Theorem 4.1 that $\int_{0}^{x} y^{\prime} / y d t \leqq G(x)$. Hence 


$$
\begin{aligned}
& {\left[\left(\int_{0}^{x}\left(p_{2}-p_{1}\right)^{1 / 2} d t\right)^{2}+\left(\int_{0}^{x} y^{\prime} / y d t\right)^{2}\right]^{1 / 2}-\int_{0}^{x} y^{\prime} / y d t} \\
& \quad \geqq\left[H^{2}(x)+G^{2}(x)\right]^{1 / 2}-G(x) \geqq \beta H(x)
\end{aligned}
$$

for some $\beta>0$ and all sufficiently large $x$. Thus the assertion is a consequence of Theorem 2.2. For the second assertion set $u_{0}=z_{0} / y_{0}$. Then $u_{0}^{\prime \prime}+2\left(y_{0}^{\prime} / y_{0}\right) u_{0}^{\prime}-\left(p_{2}-p_{1}\right) u_{0}=0$ and $\int_{0}^{x} y_{0}^{\prime} / y_{0} d t \geqq-G(x)$ for all sufficiently large $x$. Hence

$$
\left[\left(\int_{0}^{x}\left(p_{2}-p_{1}\right)^{1 / 2} d t\right)^{2}+\left(\int_{0}^{x} y_{0}^{\prime} / y_{0} d t\right)^{2}\right]^{1 / 2}+\int_{0}^{x} y_{0}^{\prime} / y_{0} d t \geqq \beta H(x)
$$

for some $\beta>0$ and all sufficiently large $x$ and the second assertion follows from Theorem 3.2.

A variant of the above concerned with exponential growth is the following.

TheOREM 5.2. Let $G$ and $H$ be as in Theorem 5.1 and suppose (a) and (b) are satisfied. If $H^{2}(x) \geqq K x(x+G(x))$ for some positive $K$, then

$$
z(x) / y(x) \geqq e^{\beta x} ; z_{0}(x) / y_{0}(x) \leqq e^{-\beta x}
$$

for some $\beta>0$ and all sufficiently large $x$.

Proof. $\left(\int_{0}^{x}\left(p_{2}-p_{1}\right)^{1 / 2} d t\right)^{2}+\left(\int_{0}^{x} y^{\prime} / y d t\right)^{2} \geqq H^{2}(x)+G^{2}(x) \geqq[\beta x+$ $G(x)]^{2}$ for some $\beta>0$ and all sufficiently large $x$. Thus the first assertion is a consequence of Theorem 2.2 as in the previous theorem. The second assertion follows similarly from Theorem 3.2.

Choosing $G$ and $H$ to be multiples of $x$ in either theorem yields a slightly more general result than that mentioned in the introduction.

CorollaRy 5.3. If $\int_{0}^{x}\left|p_{1}\right| d t \leqq M x$ for some $M$, and if

$$
\liminf _{x \rightarrow \infty} \int_{0}^{x}\left(p_{2}-p_{1}\right)^{1 / 2} d t / x>0 \text {, }
$$

then

$$
z(x) / y(x) \geqq e^{\beta x} ; z_{0}(x) / y_{0}(x) \leqq e^{-\beta x}
$$

for some $\beta>0$ and all $x$ greater than some $x_{0}$.

If $p_{1}$ and $p_{2}$ are nonnegative, then an application of Schwarz's lemma shows that (b) in Theorem 5.1, is in the presence of the other 
hypotheses of that theorem, equivalent to the in general more restrictive

$$
\text { ( b') } \lim \inf _{x \rightarrow \infty} \int_{0}^{x} \sqrt{p_{2}}-\sqrt{p_{1}} d t / H(x)>0 .
$$

The same is then true of Corollary 5.3. From the form of Theorems 2.2 and 3.2 when $r=0$ one might expect this condition to be more closely related than (b) to the behavior of $z / y$. Our final theorem may also be viewed in this way, for it implies that $z / y$ can fail to increase exponentially even when $p_{2}-p_{1}$ is bounded away from 0 provided that $\sqrt{p_{2}}-\sqrt{p_{1}}$ approaches 0 .

THEOREM 5.5. Suppose that $p_{1}$ and $p_{2}$ are nonnegative locally integrable functions such that $\left|p_{2}-p_{1}\right| \leqq M$ for some $M$ and $\sqrt{p_{2}}-\sqrt{p_{1}} \rightarrow 0$ as $x \rightarrow \infty$. Then for any positive nonprincipal solutions $y$ and $z$ of (1) and (2),

$$
(\log z / y)^{\prime} \longrightarrow 0 \text { as } x \longrightarrow \infty \text {. }
$$

It then follows immediately that for any positive $\alpha$,

$$
\log (z(x) / y(x))-\alpha x \longrightarrow-\infty
$$

as $x \rightarrow \infty$ and so, exponentiating, $[z(x) / y(x)] e^{-\alpha x} \rightarrow 0$ as $x \rightarrow \infty$.

Proof. We may assume $p_{2} \geqq p_{1}$, for the hypotheses are still valid for the functions $\min \left(p_{1}, p_{2}\right)$ and $\max \left(p_{1}, p_{2}\right)$ and the quotient of nonprincipal solutions of the equations with $p_{1}$ and $p_{2}$ replaced by these functions is greater than $z / y$. We shall for the present assume further that $p_{1}(x) \geqq 1$ for all $x$.

It suffices to extablish the theorem for the solutions $y$ and $z$ of (1) and (2) with $y(0)=y^{\prime}(0)=z(0)=z^{\prime}(0)=1$. Set $u=z^{\prime} / z$ and $v=y^{\prime} / y$ so that $u^{\prime}=p_{2}-u^{2}$ and $v^{\prime}=p_{1}-v^{2}$. Note that $p_{1} \geqq 1$ implies $v \geqq 1$, for if $v\left(x_{0}\right)<1$ then $v\left(x_{1}\right)<1, v^{\prime}\left(x_{1}\right)<0$ for some $x_{1} \in\left(0, x_{0}\right)$, and this is impossible. Similarly $u \geqq 1$. Finally, set $w=u-v$. Then

$$
w^{\prime}=p_{2}-p_{1}-(u+v) w ; w(0)=0 .
$$

Let $\varepsilon>0$ be given. Choose $c<1$ so that $K=c^{2} /\left(1-c^{2}\right)$ satisfies $M K<\varepsilon / 3$. Choose $x_{0}$ so that $\sqrt{p_{2}(x)}-\sqrt{p_{1}(x)}<c \varepsilon / 3$ for $x \geqq x_{0}$. Repeating the argument in the proof of Theorem 2.1 for the equation $u^{\prime}=p_{2}-u^{2}$ on the interval $[t, \infty)$ yields that $\int_{t}^{x} u d s \geqq c \int_{t}^{x} \sqrt{p_{2}} d s$ for all $x \geqq t$ not in a set $E_{c, t}$ such that $m\left(E_{c, t}\right) \leqq c^{2} / u(t)\left(1-c^{2}\right) \leqq K$. Hence for any $x \geqq t+K$ there is some $X \in[x-K, x]$ such that

$$
\int_{t}^{x} u d s \geqq \int_{t}^{x} u d s \geqq c \int_{t}^{x} \sqrt{p_{2}} d s \geqq c \int_{t}^{x-K} \sqrt{p_{2}} d s .
$$


Similarly $\int_{t}^{x} v d s \geqq c \int_{t}^{x-K} \sqrt{p_{1}} d s$ whenever $x-K \geqq t$.

For all $x \geqq x_{0}+K$ we have from (16) that

$$
\begin{aligned}
w(x) & =\int_{0}^{x} \exp \left(-\int_{t}^{x} u+v d s\right)\left(p_{2}(t)-p_{1}(t)\right) d t \\
& =\int_{0}^{x_{0}}+\int_{x_{0}}^{x-K}+\int_{x-K}^{x}=I_{1}+I_{2}+I_{3} .
\end{aligned}
$$

Since $u+v \geqq 2$,

$$
I_{1} \leqq M x_{0} \exp \left(-\int_{x_{0}}^{x} u+v d s\right) \leqq M x_{0} \exp \left(-2\left(x-x_{0}\right)\right)<\varepsilon / 3
$$

for all $x$ greater than some $x_{1}$.

By the choice of $x_{0}$ we have next

$$
\begin{aligned}
I_{2} & \leqq(c \varepsilon / 3) \int_{x_{0}}^{x-K} \exp \left(-c \int_{t}^{x-K} \sqrt{p_{2}}+\sqrt{p_{1}} d s\right)\left(\sqrt{p_{2}(t)}+\sqrt{p_{1}(t)}\right) d t \\
& =(c \varepsilon / 3)(1 / c) \exp \left(-c \int_{t}^{x-K} \sqrt{p_{2}}+\sqrt{p_{1}} d s\right) \int_{x_{0}}^{x-K}<\varepsilon / 3 .
\end{aligned}
$$

Finally, by the choice of $K, I_{3} \leqq K M<\varepsilon / 3$. Hence $w(x)<\varepsilon$ for all $x \geqq x_{1}$. Also $p_{2} \geqq p_{1}$ and $u(0)=v(0)$ implies $w=u-v \geqq 0$. Since $\varepsilon$ was arbitrary we have that $w=u-v=(\log z / y)^{\prime} \rightarrow 0$ as $x \rightarrow \infty$ and the theorem is proved when $p_{1} \geqq 1$.

Now let $p_{1}$ and $p_{2}$ be as in the statement of the theorem and consider the functions $y_{1}$ and $z_{1}$ such that $y_{1}(0)=y_{1}^{\prime}(0)=z_{1}(0)=z_{1}^{\prime}(0)=1$ and

$$
\begin{aligned}
& y_{1}^{\prime \prime}-\left(p_{1}+1\right) y=0 \\
& z_{1}^{\prime \prime}-\left(p_{2}+1\right) z=0
\end{aligned}
$$

Note that $\left(\sqrt{p_{2}+1}-\sqrt{p_{1}+1}\right)\left(\sqrt{p_{2}+1}+\sqrt{p_{1}+1}\right)=p_{2}-p_{1}=$ $\left(\sqrt{p_{2}}-\sqrt{p_{1}}\right)\left(\sqrt{p_{2}}+\sqrt{p_{1}}\right)$ so that $\left|\sqrt{p_{2}+1}-\sqrt{p_{1}+1}\right| \leqq\left|\sqrt{p_{2}}-\sqrt{p_{1}}\right|$. Hence the special case already proved can be applied to $z_{1} / y_{1}$.

Let $y$ and $z$ be the solutions of (1) and (2) such that

$$
y(0)=y^{\prime}(0)=z(0)=z^{\prime}(0)=1 \text {. }
$$

Then $w=y^{\prime} / y$ and $w_{1}=y_{1}^{\prime} / y_{1}$ satisfy $w^{\prime}=p_{1}-w^{2}, w_{1}^{\prime}=p_{1}+1-w_{1}^{2}$, and $w(0)=w_{1}(0)$. Also $(w+1)^{\prime}=p_{1}-(w+1)^{2}+1+2 w \geqq p_{1}+1-$ $(w+1)^{2}$. Hence $y^{\prime} / y \leqq y_{1}^{\prime} / y_{1} \leqq y^{\prime} / y+1$.

Set $s=z / y$ and $s_{1}=z_{1} / y_{1}$. Then

$$
\begin{aligned}
& s^{\prime \prime}+2\left(y^{\prime} / y\right) s^{\prime}-\left(p_{2}-p_{1}\right) s=0 \\
& s_{1}^{\prime \prime}+2\left(y_{1}^{\prime} / y_{1}\right) s_{1}^{\prime}-\left(p_{2}-p_{1}\right) s_{1}=0
\end{aligned}
$$


and by the proof so far we know $\left(\log s_{1}\right)^{\prime}=s_{1}^{\prime} / s_{1} \rightarrow 0$ as $x \rightarrow \infty$. Now $r=s / s_{1}$ is an increasing solution of

$$
r^{\prime \prime}+2\left[y^{\prime} / y+s_{1}^{\prime} / s_{1}\right] r^{\prime}-2\left(y_{1}^{\prime} / y_{1}-y^{\prime} / y\right)\left(s_{1}^{\prime} / s_{1}\right) r=0
$$

since $r(0)=1, r^{\prime}(0)=0$ and the coefficient of $r$ is nonnegative. Also $r^{\prime} / r=s^{\prime} / s-s_{1}^{\prime} / s_{1}$. Thus to establish that $(\log z / y)^{\prime}=s^{\prime} / s \rightarrow 0$ it remains, finally, only to verify that if $f$ and $g$ are nonnegative functions such that $g \rightarrow 0$ then every increasing solution of $y^{\prime \prime}+f y^{\prime}-g y=0$ satisfies $y^{\prime} / y \rightarrow 0$. To see this note that $w=y^{\prime} / y$ satisfies $w^{\prime}+w^{2}=$ $g-f w \leqq g$. It is well-known (and easy to see) that if $g \rightarrow 0$ then every solution of $u^{\prime}+u^{2}=g$ on $[0, \infty)$ does also. If $u$ is the solution with $u(0)=w(0)$, then $0 \leqq w \leqq u$ so that $y^{\prime} / y=w$ does approach 0 and the proof of Theorem 5.5 is complete.

\section{REFERENCES}

1. W. A. Coppel, Stability and Asymptotic Behavior of Differential Equations, D. C. Heath and Company, Boston, 1965.

2. G. H. Hardy, J. E. Littlewood, and G. Polya, Inequalities, Second edition, Cambridge University Press, Cambridge, 1952.

3. P. Hartman, Ordinary Differential Equations, John Wiley \& Sons, New York, 1964.

4. T. T. Read, Exponential estimates for solutions of $y^{\prime \prime}-q^{2} y=0$, Proc. Amer. Math. Soc., 45 (1974), 332-338.

5. - On the limit point condition for polynomials in a second order differential expression, J. London Math. Soc., (2), 10 (1975), 357-366.

Received June 18, 1975 and in revised form December 19, 1975.

Chalmers University of Technology, S-402 20 Goteborg 5, Sweden AND

Western Washington State College

Current address: Western Washington State College 



\section{PACIFIC JOURNAL OF MATHEMATICS}

EDITORS

RICHARD ARENS (Managing Editor)

University of California

Los Angeles, California 90024

R. A. BEAUMONT

University of Washington

Seattle, Washington 98105
J. DugundJI

Department of Mathematics

University of Southern California

Los Angeles, California 90007

D. Gilbarg and J. Milgram

Stanford University

Stanford, California 94305

\section{ASSOCIATE EDITORS}

E. F. BECKENBACH

B. H. NeumanN

F. WOLF

K. YosHIDA

\section{SUPPORTING INSTITUTIONS}

UNIVERSITY OF BRITISH COLUMBIA

UNIVERSITY OF SOUTHERN CALIFORNIA

CALIFORNIA INSTITUTE OF TECHNOLOGY

UNIVERSITY OF CALIFORNIA

STANFORD UNIVERSITY

UNIVERSITY OF TOKYO

MONTANA STATE UNIVERSITY

UNIVERSITY OF UTAH

UNIVERSITY OF NEVADA

WASHINGTON STATE UNIVERSITY

NEW MEXICO STATE UNIVERSITY

UNIVERSITY OF WASHINGTON

OREGON STATE UNIVERSITY

UNIVERSITY OF OREGON

OSAKA UNIVERSITY

AMERICAN MATHEMATICAL SOCIETY
NAVAL WEAPONS CENTER

Printed in Japan by International Academic Printing Co., Ltd., Tokyo, Japan 


\section{Pacific Journal of Mathematics}

\section{Vol. 63, No. $1 \quad$ March, 1976}

Ralph Artino, Gevrey classes and hypoelliptic boundary value problems ....... 1

B. Aupetit, Caractérisation spectrale des algèbres de Banach commutatives .... 23

Leon Bernstein, Fundamental units and cycles in the period of real quadratic

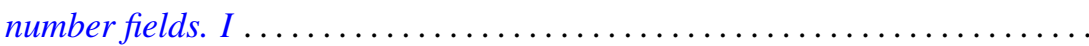

Leon Bernstein, Fundamental units and cycles in the period of real quadratic number fields. II.................................... 63

Robert F. Brown, Fixed points of automorphisms of compact Lie groups ........

Thomas Ashland Chapman, Concordances of noncompact Hilbert cube

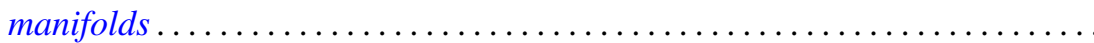

William C. Connett, V and Alan Schwartz, Weak type multipliers for Hankel

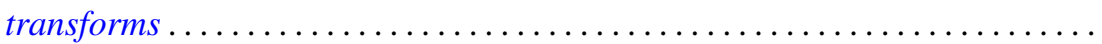

John Wayne Davenport, Multipliers on a Banach algebra with a bounded approximate identity .....................................

Gustave Adam Efroymson, Substitution in Nash functions ................ 137

John Sollion Hsia, Representations by spinor genera ..................

William George Kitto and Daniel Eliot Wulbert, Korovkin approximations in

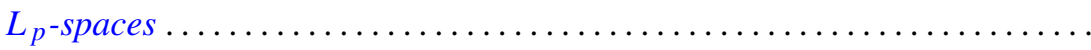

Eric P. Kronstadt, Interpolating sequences for functions satisfying a Lipschitz. condition ...........................................

Gary Douglas Jones and Samuel Murray Rankin, III, Oscillation properties of certain self-adjoint differential equations of the fourth order...

Takaŝi Kusano and Hiroshi Onose, Nonoscillation theorems for differential

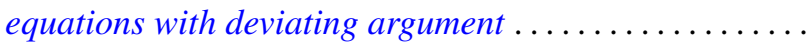

David C. Lantz, Preservation of local properties and chain conditions in commutative group rings. ...

Charles W. Neville, Banach spaces with a restricted Hahn-Banach extension property....

Norman Oler, Spaces of discrete subsets of a locally compact group ...

Robert Olin, Functional relationships between a subnormal operator and its minimal normal extension.

Thomas Thornton Read, Bounds and quantitative comparison theorems for nonoscillatory second order differential equations ...... .

Robert Horace Redfield, Archimedean and basic elements in completely distributive lattice-ordered groups...

Jeffery William Sanders, Weighted Sidon sets

Aaron R. Todd, Continuous linear images of pseudo-complete linear topological spaces.

J. Jerry Uhl, Jr., Norm attaining operators on $L^{1}[0,1]$ and the Radon-Nikodým property.

William Jennings Wickless, Abelian groups in which every endomorphism is a left multiplication. 